\title{
Tangence
}

\section{Stratégies de lecture dans le roman contemporain}

\section{Max Roy}

Numéro 39, mars 1993

La fiction postmoderne

URI : https://id.erudit.org/iderudit/025754ar

DOI : https://doi.org/10.7202/025754ar

Aller au sommaire du numéro

Éditeur(s)

Tangence

ISSN

0226-9554 (imprimé)

1710-0305 (numérique)

Découvrir la revue

Citer cet article

Roy, M. (1993). Stratégies de lecture dans le roman contemporain. Tangence, (39), 76-88. https://doi.org/10.7202/025754ar d'utilisation que vous pouvez consulter en ligne.

https://apropos.erudit.org/fr/usagers/politique-dutilisation/ 


\section{Stratégies de lecture dans le roman contemporain Max Roy}

Dans son bilan de la "critique savante des années quatrevingt " sur la littérature québécoise, Jacques Allard constate: «L'année 1960 constitue pour beaucoup le point pivotal; avant, c'est moderne (depuis 1895 ou 1900); après, c'est postmoderne " 1 . Il renvoie, en particulier, à l'ouvrage que Janet $M$. Paterson a consacré à des Moments postmodernes dans le roman québécois ${ }^{2}$. Après avoir tracé brièvement l'histoire de la notion de postmoderne, l'auteure de cette étude ne manque pas de signaler les difficultés de son usage dans. le contexte littéraire québécois. Le terme, du reste, n'apparaît guère avant le milieu des années quatre-vingt dans la critique francophone ${ }^{3}$. La question élémentaire se pose: "Le roman postmoderne existe-t-il au Québec?"4.

De fait, le paradigme postmoderne, si l'on accepte ses postulats, est une façon de réindexer des ouvres jugées jusqu'ici originales, audacieuses ou plus généralement modernes, comme des romans de Bessette, Aquin ou Godbout. Cette relecture autorise un classement des cuvres sous des procédés récurrents et sous des rubriques inspirées des théories de la littérature actuellement en vogue 5 .

1 Jacques Allard, Traverses, Montréal, Boréal, coll. ^Papiers collés», 1991, p. 94.

2 Janet $M$. Paterson, Moments postmodernes dans le roman québécois, Ottawa, Presses de l'Université d'Ottawa, 1990, 126 p.

3 S'il fallait noter quelques exceptions significatives, on retiendrait une remarque de Laurent Mailhot (*Bibliothèques imaginaires: le livre dans quelques romans québécois*, Etudes françaises, vol. XVIII, n 3, 1983, p. 93) à propos de Les grandes marées de Poulin. C'est, à son avis, *un livre que l'on peut qualifier de "postmoderniste", en ce sens qu'il dépasse à la fois le réalisme traditionnel et la modernité récente*.

4 Janet M. Paterson, op. cit., p. 5.

5 Évoquant le contexte de la Révolution tranquille, Paterson peut écrire: ‘ À ce moment-là, on ne se doutait guère que de nombreux romans écrits à partir de 1960 - romans qui contestaient les anciens systèmes idéologiques - seraient un jour appelés "postmodernes". Elle ajoute aussitôt: - Pourtant, en se situant dans une optique lyotardienne, on peut affirmer que c'est bien une crise postmoderne qui a traversé - et qui traverse toujours - la société québécoise* (Janet $M$. Paterson, ibid., p. 17). 
Si elle a valeur de classement, ainsi qu'il en allait jadis des catégories de l'histoire littéraire, la notion de postmoderne n'a pas davantage une fonction opératoire. Pour s'en faire une idée juste, quel point de vue critique faut-il adopter? Celui de la philosophie, le plus souvent convoqué, a certainement le mérite d'inscrire la problématique du roman québécois dans une réflexion générale sur les conditions de la pensée, du savoir et de l'agir qui échappe aux contingences historiques, géographiques ou politiques. La notion, pourtant, ne s'applique pas indifféremment à toute manifestation de la pensée contemporaine, mais elle est attribuable à certains usages. Ce sont précisément, en ce qui nous concerne, des usages de l'écriture et de la lecture. Au delà de tendances communes et de visées transculturelles, il y aurait donc plusieurs pratiques postmodernes parmi lesquelles des pratiques littéraires. Ainsi, Janet $M$. Paterson est tout à fait justifiée de désigner par le pluriel l'objet de son étude: ce sont des "moments postmodernes" distincts. "A mesure que l'écriture postmoderne se diversifie, écritelle, il devient de plus en plus évident que la convention postmoderne " n'est ni un dogme, ni une forme figée " 6 . Cette convention, du reste, ne semble pas promise à un grand avenir d'après Paterson, qui va jusqu'à écrire: «Il est tout à fait probable qu'avant la fin de notre siècle, le roman postmoderne sera considéré comme une convention littéraire dépassée. [...] On parlera, sans doute, de cette esthétique comme on parle aujourd'hui du surréalisme ou même du nouveau roman." 7 . Cette prédiction a de quoi surprendre. Elle laisse entendre que les paramètres du postmoderne peuvent bien être fixés mais que leur prolongement est impossible, qu'une transformation radicale, plutôt, est à prévoir, éventuellement sous le signe d'une reconstruction.

Du côté du roman, on peut observer à l'heure actuelle une cohabitation de tendances diverses où des procédés d'écriture modernes voisinent des procédés nettement plus traditionnels, où l'accent mis sur le langage n'interdit pas des effets de références réalistes et où des "histoires de papier" renferment ce qui semble être des emprunts à la vie réelle pour composer, le cas échéant, une révision de l'Histoire. Lorsqu'il fait prévaloir l'intrigue et le divertissement, ce "retour du lisible" est peut-être bien suspect ${ }^{8}$.

$6 \quad$ Ibid., p. 84.

7 Ibid., p. 93.

8 Régine Robin, *Le retour du lisible*, Spirale, n 68, p. 2-3. 
Cette nouvelle lisibilité signifie-t-elle pour autant un retour du lecteur? Celui-ci n'était-il pas, justement, inscrit dans des pratiques postmodernes?

Cette question de la lecture en rapport avec des pratiques contemporaines déplace la problématique de la fiction littéraire, de sa mise en ceuvre à son interprétation. Il est trop tôt assurément pour mesurer toutes les implications de ces pratiques et pour les inscrire dans une histoire de la lecture littéraire au Québec. Je me propose simplement de faire quelques observations sur les stratégies de lecture mises en place dans des romans singuliers, en retenant surtout des romans québécois récents.

Pour étudier la question de la lecture littéraire, il faut mettre de côté les conditions élémentaires d'une absorption (attention, mémoire, vocabulaire, etc.) qui verseraient les incompréhensions et les "mésinterprétations" au compte d'une rupture accidentelle de contact. On peut partir de la place occupée par le lecteur pour définir des modes de manifestation. Ainsi, le lecteur réel se trouve bien évidemment à l'extérieur du livre: selon une formule consacrée, il est dans le hors-texte. Que ce soit pour des raisons personnelles ou professionnelles, ce lecteur se transforme parfois en un lecteur critique et il se manifeste dans un discours métatextuel $^{9}$. Il arrive aussi que le lecteur fasse explicitement partie du livre. Il est pris en charge dans un paratexte ou encore il est intégré à la fiction, à titre de personnage, et il prend alors un statut textuel. Ces deux positions ne vont pas de soi. Elles supposent des stratégies particulières correspondant à des contrats de lecture et à des modes de connaissance.

D'entrée de jeu, un livre de François Barcelo oblige le lecteur à se questionner. Cet ouvrage intitulé $\mathrm{Aaa}, \mathrm{A} a \mathrm{~h}, \mathrm{Ha}$ ou les amours malaisées ${ }^{10}$ arbore une indication générique au pluriel: « romans ". L'organisation de la matière textuelle est ainsi annoncée, comme s'il s'agissait de plusieurs récits ou nouvelles. On découvre vite que les trois "romans" sont dispersés dans l'ensemble du livre de

9 Il se trouve, en l'occurrence, des relectures d'œuvres des décennies 1960 et 1970 qui mettent en évidence des questions d'intertextualité, dont on a fait une caractéristique de la fiction postmoderne. Je n'insisterai pas ici sur les formes et les fonctions du métatexte littéraire.

10 François Barcelo, Aaa, Aâb, Ha ou les amours malaisées. Romans, Montréal, l'Hexagone, 256 p. 
Barcelo et que leur compréhension, le cas échéant, ne sera pas indifférente au fait de cette dissémination. La contiguité ou la concurrence des épisodes romanesques n'est certes pas sans conséquence. Cet ouvrage, qui a peu retenu l'attention de la critique, est une fable fantastique au ton léger. Par la description que l'auteur en donne, on devine immédiatement son caractère humoristique. Mais, plus encore qu'un résumé du contenu, la préface auctoriale de $\mathrm{Aa}, \mathrm{A} a \mathrm{~b}, \mathrm{Ha}$ ou les amours malaisées est une invitation à une libre aventure de lecture.

Il y a plusieurs manières de lire ce livre.

La première est, bien entendu, celle dans laquelle il est présenté, en commençant par le début et en terminant par la fin.

Mais on peut aussi bien lire d'abord tous les chapitres sous-titrés "Aaa", puis tous les chapitres "Aâh", et terminer par les chapitres "Ha". Â moins qu'on ne préfère commencer par "Aâh" ou par "Ha . Il faut toutefois garder pour la fin le chapitre «Aaa, Aâh et $\mathrm{Ha}$.

Que faire du premier chapitre - «A, Une et Ahune "? Là encore, plusieurs possibilités s'offrent au lecteur un tant soit peu aventureux: le lire avant le reste (la plus logique, recommandée par l'auteur), dès que le besoin s'en fera sentir (la plus raisonnable et la plus fantaisiste à la fois), ou même ne pas le lire du tout (la plus rapide) ${ }^{11}$.

Pour bien marquer la multiplication de ces possibilités, Barcelo ajoute:

On en arrive donc à des centaines, peut-être des milliers, sinon des millions de combinaisons possibles. Et confier au hasard l'ordre de lecture (en mettant, par exemple, les différentes possibilités sur des bouts de papier, dans un chapeau) n'est pas

11 L'auteur écrit notamment: *Pour guider toute personne désireuse de faire un choix rationnel, précisons que les chapitres "Aaa" sont un peu écologistes, vaguement rétro, plutôt amusants pour quiconque apprécie l'humour discret [...].

Les chapitres "Aâh" ressemblent à une satire sociale bourrée de clés, sauf que les clés sont dans plusieurs cas tout à fait introuvables, peut-être mêrne inexistantes, bien qu'il en existe parfois. L'humour y est gros et lourd, la morale en est boiteuse ou absente, et on peut tout aussi aisément détester ces chapitres que les préférer aux autres.

Les chapitres $\cdot \mathrm{Ha}$ × sont les moins déroutants et présentent tantôt une fillette inquiétante, tantôt un adulte découvrant l'érotisme à un âge avancé, le tout sur fond de menace de guerre nucléaire.x (ibid., p. 14). 
moins sensé que de confier ce soin à un écrivain trop impliqué dans son cuvre ou à un éditeur bourré de préjugés sur les goûts des lecteurs ${ }^{12}$.

Cette préface de l'auteur, intitulée "Conseils de lecture", est tout le contraire d'une programmation rigoureuse. La lecture à laquelle elle invite n'est pas tenue de suivre un cheminement singulier ou de respecter un code qui serait fourni en appendice à la fiction. À l'encontre d'un système de règles ou des instructions qui accompagneraient un jeu intellectuel, on proclame la liberté de la lecture. Le livre est un objet manipulable et lisible de multiples manières et ses significations restent foncièrement imprévisibles. La lecture concrétise, en quelque sorte, le caractère indéterminé du sens ${ }^{13}$. Elle confirme qu'un texte est une "véritable machine à susciter des interprétations " 14 .

Les conséquences de ces positions sur la lecture pourraient être nombreuses et heureuses. Je ne mettrai pas en doute la bonne foi de l'auteur ni celle du lecteur qui aura compris qu'il peut explorer à loisir cette fable contemporaine. Bien entendu, la pratique de ce mode de lecture est incontrôlable. La seule affirmation de sa possibilité signifie un contrat de lecture très souple et un statut variable du texte, selon ses usages. Non seulement est démenti un caractère absolu de l'écriture ou de la "mise en texte", mais de plus la pertinence d'un principe d'ordre dans une stratégie cognitive est tout simplement niée. Cette négation peut

12 Ibid., p. 13.

13 L'ouvrage s'ouvre, par ailleurs, sur un "avertissement " de l'auteur qui concerne le statut de la fiction, plus exactement les rapports entre la représentation et la référence extérieure:

-Il existe, dans certaines parties de l'univers représenté ici, des objets, des animaux et des êtres qui n'ont aucune ressemblance avec ceux qui existent sur la planète qu'habitent selon toute probabilité la plupart des lecteurs de ce récit. À ces objets, animaux et êtres divers, nous avons prêté un nom descriptif qui devrait donner une notion satisfaisante de leur apparence ou de leur fonction.

Mais cette histoire met aussi en scène des objets, des animaux et d'autres êtres qui présentent quelque ressemblance avec des objets, animaux ou autres êtres vivant sur la planète où sont sans doute la majorité de ses lecteurs immédiats. Nous avons préféré leur donner le nom des sujets les plus ressemblants aux yeux de ceux-ci, de façon à clarifier et alléger le récit. Le lecteur ou la lectrice devra toutefois se garder d'interpréter ces noms comme une représentation exacte de ce qu'ils désignent normalement. (ibid., p. 11).

14 Umberto Eco, Les limites de l'interprétation, Paris, Grasset, p. 148. 
bien n'être qu'une fantaisie de l'auteur ou l'expression d'un dessein ironique. Elle met en évidence le rôle du lecteur.

Ce rôle est rendu explicite, en tout cas, alors qu'il était sousentendu sinon modeste dans une esthétique plus traditionnelle et dans une lecture linéaire. Entendons qu'il se passe autre chose ici qu'un appel convenu à la bienveillance ou qu'une figuration du lecteur-type dans le champ de la fiction. De fait, le lecteur reste hors-champ, bien qu'il soit interpellé textuellement. Paradoxalement peut-être, s'il est maintenu à l'extérieur de la fiction, on lui concède le droit d'en disposer à son gré, c'est-à-dire de la transformer en connaissance. Cette transformation repose sur une stratégie chaque fois renouvelée. Ce n'est donc pas par un effet de compétence préalable que le texte proposé devient une fiction déchiffrable, mais c'est par sa prise en charge - sa reconstruction, le cas échéant qu'il fait advenir une compétence. Toute littéraire, sans doute, cette compétence est le produit d'une performance de lecture.

Dans l'exemple précité, la problématique de la lecture du récit est inscrite en clair dans ce qu'il est convenu d'appeler, avec Genette, un "péritexte"15. Cependant, le péritexte ne se réduit pas aux discours d'accompagnement des œuvres littéraires qui prennent la forme d'avant-propos, de préface ou de postface. Il inclut également les titres, les intertitres, les illustrations et, s'il y a lieu, les notes d'éditeur qui constituent un discours parallèle et dont l'importance est variable. Par exemple, en faisant l'analyse du péritexte multiformel d'un roman de Nabokov (Pale Fire ${ }^{16}$ ), un critique a été conduit à le qualifier de "parasitaire " parce que référentiellement opaque. De plus, pouvait-il ajouter,

Contrairement à la quasi totalité des récits, ce texte ne doit pas être lu linéairement mais, comme Kinbote le suggère dans son introduction, d'une manière bien particulière. Au lecteur, il est conseillé de consulter les "notes, réunies en un commentaire soutenu", qui sont situées après le texte du poème, puis de les relire en même temps qu'il lit le poème lui-même et, ensuite, « de les consulter une troisième fois pour obtenir une vue d'ensemble. . Pour le lecteur qui ne résiste pas à la tentation de tricher, cela représente environ trois discours composés par un texte qui est, en principe, unique ${ }^{17}$.

15 Gérard Genette, Seuils, Paris, Seuil, coll. «Poétique „, 1987.

16 Vladimir Nabokov, Pale fire, New York, Putnam.

17 John Pier, "Pragmatique du paratexte et signification *, Études littéraires, vol. XXI, no 3 , hiver $1988-1989$, p. 115-116 
82

Si l'ordre de ces activités est jugé pertinent, il représente, en fait, une forte contrainte de lecture. Un jeu paratextuel approchant est à l'œuvre dans Trou de mémoire d'Hubert Aquin ${ }^{18}$. Pour Janet M. Paterson, c'est " incontestablement le roman qui s'inscrit le plus fidèlement dans la poétique postmoderne. Comme Pale Fire de Nabokov, il est en fait exemplaire du genre "19. Elle observe, en effet, que les nombreuses notes éditoriales fictives correspondent à une "structure oxymorique, celle-là même qui permet de juxtaposer des sens réputés incompatibles " ${ }^{20}$. Elle affirme, par ailleurs, que "l'annotation fictive est une stratégie textuelle qui, par le biais de la mouvance et de l'altérité, permet de remettre en question les principes d'unité discursive et d'homogénéité des codes de lecture "21. De pareils effets de rupture et d'hétérogénéité s'obtiennent aussi par le fragment et le collage. Dans un roman à forte teneur autobiographique, par exemple, Régine Robin ${ }^{22}$ nous fait partager l'expérience de "l'étrangeté ". La Québécoite ne se contente pas de dire, elle fait voir des objets et des discours à apprivoiser en reproduisant, entre autres choses, des horaires de la programmation télévisée et des menus de restaurant.

Un récit composé de fragments comporte d'inévitables interstices, comme des moments laissés à la réflexion. On aime à penser que c'est une place faite à l'intelligence. Une lecture particulièrement active est requise, suppléant une rationalité de discours. Plutôt que d'imposer une interprétation totalisante et une lecture captive, l'œuvre est un objet d'expérience. À une lecture captive s'oppose théoriquement une lecture libre. Il faut la définir, néanmoins, comme une recherche de cohérence ou un programme de sens. C'est, bien sûr, sur le plan de la sélection des informations et du parcours utilisé que peut se définir librement une stratégie de lecture. L'analyse fait vite apparaître, pourtant, d'autres possibilités d'échapper à des contraintes du texte, parmi lesquelles l'insouciance et l'errance seraient des figures négatives. L'application et l'utilisation, en revanche, seraient des manières positives d'un investissement de la part du sujet lecteur - mais qui ne seraient pas moins suspectes si l'on songe aux fins poursuivies par une lecture critique.

18 Hubert Aquin, Trou de mémoire, Montréal, Le Cercle du livre de France, 1968.

19 Janet M. Paterson, op. cit., p. 43.

20 Ibid., p. 47.

21 Ibid., p. 48.

22 Régine Robin, La Québécoite, Montréal, Québec/Amérique, 1983. 
À cet égard, la lecture de Pale Fire ou de Trou de mémoire parait réclamer une application peu commune, sinon une attitude de spécialiste. Le lourd arsenal de notes, qui fait inévitablement penser à l'édition critique, contribue à une dispersion du sens. Celui-ci ne peut pas être atteint par une lecture linéaire et progressive, mais il s'accomplit dans un aller-retour de la lecture, du texte de fiction aux pseudo-notes de l'éditeur ou même, pour utiliser un mot à la mode, par un "zapping" dans l'espace du texte. Cela confirme, bien sûr, qu'il n'y a pas un sens unique ou un seul parcours de lecture. Partant, la question n'est pas de savoir si les lectures possibles sont équivalentes en termes de rendement (compréhension, satisfaction, utilisation, etc.) ou si elles sont correctes par rapport à une intentionnalité de l'auteur. Ce n'est pas la vérité comme issue de la lecture qui importe, car elle est toujours contestable, mais c'est la validité du parcours, c'est-à-dire son caractère approprié à l'objet-texte et à un objectif de lecture. Si le lecteur peut s'abandonner spontanément à la fiction et à la découverte d'une histoire, il n'empêche qu'une visée de lecture, loin d'être immuable, est portée par le texte. Elle est adaptable, forcément, sans quoi elle ne peut qu'être déçue.

Ainsi, suivant des usages actuels de la fiction, les lecteurs de romans sont parfois prévenus, par le péritexte, des possibles voies à emprunter. Ayant pris connaissance de l'avant-propos d'un roman de Pauline Harvey, par exemple, le lecteur ne peut plus ignorer que "le jeu de la narratrice à la recherche de son lecteur est un simulacre de l'auteur "23. Dans l'introduction, il apprend la volonté de la narratrice d'écrire un roman qui s'intitulerait Pitié pour les salauds!, ce qui est confirmé par le titre du premier chapitre: "Où la narratrice prend la décision d'écrire Pitié [...J. Il n'aura de cesse de savoir si ce roman aura finalement été écrit.

Sur un autre plan, un même lecteur aura peut-être compris assez tôt qu'il n'y avait pas d'issue aux histoires successives. que raconte Italo Calvino dans un livre étourdissant: Si par une nuit d'biver un voyageur ${ }^{24}$. S'il était à la recherche d'une intrigue, son intérêt se sera tourné vers l'histoire du Lecteur et de la Lectrice

23 Pauline Harvey, Pitié pour les salauds!, Montréal, l'Hexagone, 1989, 192 p.

24 Italo Calvino, Si par une nuit d'biver un voyageur, Paris, Seuil, 1981 [1979]. 
84

personnifiés dans le roman. Un lecteur avisé, probablement, aura reconnu dans les incipits des pastiches de différents genres romanesques. Mais enfin, qui aura soupçonné que la composition des chapitres numérotés de ce livre reposait sur une adaptation toute personnelle du carré sémiotique de Greimas et sur un développement par mode d'expansion ${ }^{25}$ ?

Dans les exemples précédents, le rôle du lecteur est mis en évidence de façon explicite, soit par des adresses, dans le péritexte, soit par une représentation dans la fiction elle-même. On ne compte plus les personnages de romans qui sont aussi des lecteurs. C'est un lieu commun qui correspond peut-être à une réalité d'époque et il serait tentant d'interpréter sociologiquement ce phénomène. Quoi qu'il en soit, il s'agit encore de lecteurs imaginaires, présumés ou attendus, que l'on fait intervenir dans un univers discursif ou évoluer dans la fiction. Au mieux, on présume des intentions du lecteur réel et de ses capacités d'identification. Par-delà ses possibles fonctions didactiques ou ludiques, cette figure courante du lecteur dans la fiction contemporaine met l'accent sur l'activité de lecture elle-même et elle invite à s'interroger sur la connaissance.

$\mathrm{Si}$ elle a toujours été traversée par des enjeux de pouvoir, la connaissance est d'abord une appropriation du monde. À cet égard, la lecture joue un rôle important et qui était grandement valorisé dans une culture classique. Mais le savoir ne se limite pas à une méthode. Un mode de connaissance privilégié reste, sans doute, la perception visuelle qui est tout autant à l'œuvre dans une lecture que dans le visionnement d'un film ou dans l'observation clinique. On imagine mal qu'il puisse en être autrement. Néanmoins, des positions de lecture diverses peuvent être déterminées en fonction de cette catégorie perceptive.

Disons un mot, d'abord, de la recherche d'effets sur le lecteur réel au moyen de la présentation matérielle du livre. À ce propos, les aspects graphique et iconographique d'un roman de Nicole Brossard, Le dêsert mauve 26 , attirent particulièrement l'attention. Laissons de côté la présentation extérieure ainsi que la division en chapitres. Le roman est constitué de trois ensembles bien

25. Italo Calvino, "Comment j'ai écrit un de mes livres", Actes sémiotiques, vol. VI, $\mathrm{n}^{\circ} 51$.

26 Nicole Brossard, Le désert mauve, Montréal, l'Hexagone, 1987. 
distincts, à la fois quant au contenu et quant à la mise en forme. Deux versions d'un récit possèdent chacune une couverture originale et une pagination distincte, comme pour appuyer leurs différences. La partie centrale, qui est la réflexion de la traductrice, comprend un dossier photographique d'un personnage, "l'homme long", dont la présentation reprend la forme typique d'une "chemise à dossier". On aura compris que le lecteur dispose ici, et de façon privilégiée, d'un document sur l'histoire d'un roman: sa version originale, sa traduction et les notes de la traductrice. Point n'est besoin d'insister, je pense, sur un effet référentiel et sur le renforcement par l'image du thème de la traduction. On pourrait signaler, de même, l'anonymat du personnage masculin qui est préservé tout autant dans les photographies qu'il l'était dans le texte. Il y aurait davantage à dire sur les rapports nombreux entre le texte et le paratexte matériel. Je retiens simplement, dans la perspective du lecteur, qu'une position d'observateur privilégié a été créée. On serait tenté de dire, en fermant le livre, qu'on a assisté au spectacle de la traduction. Mais, il faudrait enlever au mot "spectacle" son sens superficiel et insister sur la participation à une lecture toute personnelle, à un "corps à corps avec le livre" 27 .

Dans l'espace de la fiction également, un tel rapport entre le lecteur et le contenu peut donner lieu à une figure de spectateur. Pour en rester au domaine québécois, Neige noire ${ }^{28}$ serait un bon exemple de roman pluriel où les personnages-lecteurs sont appelés à devenir successivement acteurs et spectateurs, soit au thêatre, avec Hamlet, soit au cinéma avec la réalisation à venir d'un (pseudo-)scénario de film. Cette rencontre de genres divers, en l'occurrence, produit un déplacement de la lecture comme appropriation des signes et elle force une modification de sa visée. Ce qui est destiné à l'écran de cinéma est-il donné à lire en transparence? Fallait-il s'étonner, du reste, qu'un lecteur admiratif eût souhaité voir se concrétiser le projet de film inscrit dans le roman? Ce jeu du vrai et du faux, du plus faux encore dans l'apparemment vrai, est une caractéristique de l'écriture aquinienne. Mais il y a plus, car cette question des rapports entre la fiction et la réalité est devenue particulièrement fréquente dans la littérature québécoise.

Ibid., p. 177.

28 Hubert Aquin, Neige noire, Montréal, La presse, 1974. 
86

Prenons un dernier exemple, récent et moins connu celui-là. Un récit de la fin des années 1980 intitulé Scènes d'enfants aborde cette question d'une manière originale et pratique. Il s'agit du premier récit de Normand Chaurette ${ }^{29}$, qui s'est d'abord fait connaître comme dramaturge. Écrit dans un style simple et parfois grave, ce roman n'a rien à voir avec le caractère excentrique des romans d'Aquin ou de Godbout. Le titre renvoie directement aux pièces pour piano de Robert Schumann. La lecture est ici interprétation, au sens théâtral. Elle est mise en scène dans un espace rendu réel par une description détaillée et par une illustration ${ }^{30}$. Le canevas du récit est le suivant. Un dramaturge cherche à faire la lumière sur des événements qui ont marqué l'enfance de sa compagne et qui seraient à l'origine de sa folie. Il écrit:

Ce fameux secret que seuls des morts auraient pu révéler, je le connaissais assez bien pour me l'être inventé. Mais eux devaient me raconter leur histoire. $\hat{A}$ vrai dire, ma reconstitution du passé ne pouvait pas réellement différer d'une réalité vécue une vingtaine d'années auparavant: dans sa folie, Vanessa m'avait tout juste fourni le nombre d'éléments nécessaires à l'organisation d'un récit, et un seul. Il m'apparut si monstrueux au départ que je cherchai à en échafauder un autre, même s'il devait être cousu d'invraisemblances ${ }^{31}$.

Il souhaite, par la même occasion, récupérer une enfant dont ses beaux-parents ont obtenu la garde. (Ce sont des gens fortunés et influents - l'homme est un sénateur américain.) Afin de les confronter, il imagine une mise en scène qui l'impliquerait lui-même ainsi que deux comédiennes. À leur insu, les beaux-parents seraient appelés à jouer leur propre rôle dans ce théâtre de la vérité. C'est à la préparation de cette représentation que l'on assiste, incluant les réflexions du dramaturge et son travail d'écriture, d'une part, les répétitions avec les acteurs, d'autre part. Des extraits d'un cahier de notes et une prolepse itérative font également partie des procédés de narration. Dans l'univers du récit, la lecture se fait interprétation d'une scène inventée mais qui doit se confondre avec la réalité.

L'enjeu épistémique de ce rapport entre le théâtre et la vie réelle est, positivement, l'éclairage de la réalité par la fiction. Le

\footnotetext{
29 Normand Chaurette, Scènes d'enfants, Montréal, Leméac, 1988. 30 Le récit inclut un plan devant servir à mieux se représenter le «lieu du
crime. (ibid., p. 55).

31 Ibid., p. 15.
} 
lecteur hors-texte, s'il s'est laissé gagner par l'intrigue, compte évidemment sur la réussite de la fiction pour résoudre une énigme. Le piège est tendu mais pas seulement pour les personnages. Les comédiennes obligent l'auteur à réviser son manuscrit et même à le récrire en grande partie. Alors que l'on croit assister à la mise en ouvre du piège, c'est à une relecture que nous avons affaire.

Par le jeu concurrent des illusions, la scène imaginée, à laquelle tout lecteur confiant se serait laissé prendre, et la vérité, qu'il croirait avoir découverte en même temps que les acteurs, se révéleraient tout aussi fausses que les références inscrites dans le texte. "Mais ce n'est pas une pièce, c'est un roman, dit Gila Rogalska. On ne sait trop où commencent les faits et où ils se terminent, car le meilleur et le pire s'entremêlent " ${ }^{32}$. Au total, cette quête de la vérité est, du point de vue de la lecture du récit, le triomphe de l'incertitude. La grande scène reste à jouer et le spectateur est resté un lecteur.

Dans une perspective de lecture, cette tendance à la matérialisation, qui traduit le lisible en visible, favorise le développement de compétences multiples. C'est tantôt celle du lecteur d'éditions critiques, tantôt celle du spectateur au théâtre ou au cinéma, tantôt encore celle de l'interprète. D'autres compétences reposent, parfois, sur des modes de connaissance non institutionnels mais parfaitement légitimés dans des pratiques culturelles courantes, comme la lecture du livre illustré. La conjonction de différents genres littéraires et l'emprunt de procédés divers supposent, par ailleurs, des contrats de lecture complexes.

À travers des pratiques inédites ou hybrides qui peuvent être qualifiées de postmodernes, notamment au sens où elles utilisent à leurs propres fins des procédés modernes, la prise en charge du procès de lecture dans le texte de fiction constitue une perspective esthétique et critique séduisante parce que ses effets sont immédiatement interprétables. Du reste, lorsqu'on arrive à imposer simultanément et avec succès plusieurs types fictionnels et plusieurs contrats de lecture, c'est au prix d'une nouvelle illusion qui se fonde moins sur une confiance en la référence que sur une croyance en la communication. Une distance critique et une visée ludique sont présupposées dans cette illusion communicative. 
88

L'inclusion du lecteur dans la fiction joue, par ailleurs, un rôle heuristique qui se traduit concrètement par l'adoption de stratégies diversifiées de la connaissance et par une performance de l'interprétation. Elle pourrait bien signaler, enfin, après le doute ou le refus, une double réhabilitation de l'acte de lecture lui-même et du romanesque. 